\title{
Feedback Linearization and Optimal Control of the Kermack-McKendrick Model for the Spread of Epidemics
}

\author{
Adela Ionescu ${ }^{1}$, Mario Lefebvre $^{2^{*}}$ and Florian Munteanu ${ }^{1}$ \\ ${ }^{1}$ Department of Applied Mathematics, University of Craiova \\ Al.I. Cuza 13, Craiova 200585, Romania \\ ${ }^{2}$ Department of Mathematics and Industrial Engineering, Polytechnique Montréal \\ C.P. 6079, Succursale Centre-ville, Montréal, Québec H3C 3A7, Canada \\ Email: mlefebvre@polymtl.ca
}

\begin{abstract}
Using the feedback linearization method, a state feedback control for the two-dimensional Kermack-McKendrick model for the spread of epidemics is obtained. This form of the dynamical system is suitable to carry out a qualitative analysis of the model. An optimal control problem for a stochastic version of the model is also set up and solved explicitly in a particular instance.
\end{abstract}

Keywords: SIR model; Bailey model; dynamical systems; LQG homing; Brownian motion.

\section{Introduction}

In this paper, we consider the problem of finding a state feedback control for the two-dimensional Kermack-McKendrick model for the spread of epidemics, in order to obtain a suitable form for the analysis of this system. The results could be used both for the three-dimensional Kermack-McKendrick model and for further generalizations. A similar study for Lotka-Volterra systems was carried out by Ionescu and Munteanu in [1], [2].

Epidemiology has been and will remain an exciting area for the modern application of mathematics. Mathematical models play a vital role in analyzing the spread and control of different diseases. J. Graunt ([3]) was the first scientist to try to quantify causes of death in 1662 , and his analysis led to the foundation of modern epidemiological theory ([4]).

The first epidemiological mathematical model describing an infectious disease was proposed by the Swiss mathematician D. Bernoulli in 1760 ([5], [6]). Bernoulli studied the impact of inoculation with cowpox upon the life expectancy of the immunized population and argued for the importance of inoculation ([7], [4]). Recently, an interesting dynamical model was presented for the immune response to a pulmonary infection $([8])$.

In 1927, Kermack and McKendrick ([9]) developed infectious disease models to study the Great Plague of London for the period 1665-1666. These models served as the foundation of theoretical models in epidemiology ([10], [11]). The population is divided into three categories as follows: susceptible, infected, and recovered individuals ([9]), and the model is called the SIR model. This model of evolution of epidemics is without demography, which means that the number of individuals in the population does not change. In [12], Brauer generalized the SIR model to include births and deaths.

The nonlinear differential equations arising from SIR models are equations of the Lotka-Volterra type ([13], [11], [14], [15]). Moreover, this kind of ODE (ordinary differential equations) systems represents a starting point for the study of the human immunodeficiency virus (HIV), the transmission dynamics of tuberculosis and others infectious diseases ([10], [12], [9], [13], [7], [11]).

The study of mathematical models of epidemiology is essential in order to cover the essential aspects of infectious diseases spread and it helps public health decision makers to compare, plan, evaluate, and implement different control programs. The analysis of the systems may be used, in particular, to study the dynamics of many other models from ecology, molecular biology, ecosystems, and chemical systems. For example, a model for oxygen depletion in a sewage system could be developed ([11]).

The qualitative analysis of mathematical models associated with multi-species interactions is very important in determining long-time dynamics, together with the study of the main characteristics of 
these models. This analysis is based on the application of various notions from the theory of dynamical systems to the numerical approximation of initial value problems over long time intervals. There is a strong interplay between the theory and the computational analysis of dynamical systems $([1],[16],[17]$, [18], [19], [20]). In [1], [2], [21] a study of the Lotka-Volterra equations by geometrical tools was carried out, using the Hamilton-Poisson structures of this system of ordinary differential equations.

The feedback linearization method is an interesting and useful practical tool. This theory contains two fundamental nonlinear controller design techniques: input-output linearization and state-space linearization ([22], [23]). We can state that a variety of nonlinear controller design techniques are based on input-output linearization and that important problems remain unsolved ([24]). The approach is usually referred to as input-output linearization, or feedback linearization, and is based on concepts from the theory of nonlinear systems. The resulting controller involves the inverse of the dynamical model of the process, providing that such an inverse exists. This approach is widely used in several process control design methods that are applicable to broad classes of nonlinear control problems ([22], [23]).

The structure of the paper is the following: in Section 2, we will shortly present the mathematical models for the spread of epidemics, as formulated by Kermack (1927) and McKendrick (1932). In Section 3 , the feedback linearization principles will be described and, in Section 4, the technique will be applied to the two-dimensional Kermack-McKendrick system.

In Section 5, a stochastic version of the feedback linearization will be considered, and an optimal control problem for this stochastic system will be set up and solved explicitly in a particular instance. The problem in question is a particular LQG homing problem (see [27]). In general, this type of problem is very difficult to solve explicitly (and exactly) in two or more dimensions. Here, we will appeal to the method of similarity solutions to do so.

Finally, some conclusions and future objectives will be mentioned in Section 6 .

\section{Modeling the Kermack-McKendrick Dynamical System}

In this section, we shortly present two very important examples: the Bailey model ([9], [16], [17], [18], [19], [25]) and the classical Kermack-McKendrick model for the spread of epidemics ([9], [16], [17], [18], [19], [25]). The Bailey model is a simplified particular case of the classical Kermack-McKendrick model. Both examples represent so called variational dynamical systems, that is, dynamical systems which are described by a system of ordinary differential equations that can be written as the Euler-Lagrange equations associated to a Lagrangian $L$ ([20], [26]):

$$
\frac{d}{d t}\left(\frac{\partial L}{\partial y^{i}}\right)-\frac{\partial L}{\partial x^{i}}=0 \text { for } i=1,2, \ldots, n
$$

\subsection{The Bailey Model for the Spread of Epidemics}

In the Bailey model for the spread of an epidemic $([10])$, two classes of hosts are considered: individuals suspected of being infected, whose number is denoted by $x$, and individuals who are infected carriers, whose number we denote by $y$. Assume that the latency period and the average removal rate are zero, so that individuals who are infected carriers remain in the population during the entire epidemic, with no death, healing and immunity. In this model, it is proposed that, in unit time, the increase of the number of individuals suspected of being infected is proportional to product of their current number and the number of those infecting them. These assumptions lead us to the evolutionary dynamical system given by $([10],[25])$

$$
\left\{\begin{array}{l}
\dot{x}=-k x y \\
\dot{y}=k x y
\end{array}, k>0\right. \text {. }
$$

The model is suitable for diseases known in animal and plant populations, and also corresponds quite well to the characteristics of small populations, such as students in a class.

Let us remark that we have a conservation law: $x+y=n$, which means that $n$, the total number of individuals in the population, does not change during the evolution of the epidemic. Equations (2) can be 
written as Euler-Lagrange equations, where the Lagrangian $L$ is

$$
L=\frac{1}{2}\left(\frac{\ln y}{x} \dot{x}-\frac{\ln x}{y} \dot{y}\right)+k(x+y)
$$

and the corresponding Hamiltonian $H$ is

$$
H=\frac{\partial L}{\partial \dot{x}} \dot{x}+\frac{\partial L}{\partial \dot{y}} \dot{y}-L=-k(x+y) .
$$

\subsection{The Classical Kermack-McKendrick Model for the Spread of Epidemics}

This three-dimensional dynamical system is also known as the $S I R$ model of epidemics, where $S$ is the number of individuals suspected of being infected, $I$ is the number of infected individuals and $R$ denotes the number of individuals removed.

The classical model for the spread of epidemics was formulated by Kermack (1927) and McKendrick (1932) as follows ([9]): let us denote the size of the population by $n$ and let us divide it into three classes: the number $x$ of individuals suspected of being infected, the number $y$ of individuals who are infected carriers, and the number $z$ of infected individuals who are isolated (or removals).

For simplicity, we assume a zero latency period and that all individuals are simultaneously infected carriers that infect those suspected of being infected. Considering the previous example, we note the constant rate $k_{1}$ of disease transmission. The change in the number of infected carriers depends on the rate $k_{1}$ and also on $k_{2}$, the rate at which infected carriers are isolated. We thus have the following system $([9],[25])$ :

$$
\left\{\begin{array}{l}
\dot{x}=-k_{1} x y \\
\dot{y}=k_{1} x y-k_{2} y, k_{1}, k_{2}>0 . \\
\dot{z}=\quad k_{2} y
\end{array}\right.
$$

Notice that $x+y+z=n$; that is, the number of individuals in the population does not change. This conservation law shows us that this SIR model for the spread of epidemics is without demography. The evolution of a dynamic epidemic begins with a large population which is made up of a majority of individuals suspected of being infected and a small number of infected individuals. The initial number of isolated infected individuals is assumed to be zero. So, we can consider the subsystem ([25]):

$$
\left\{\begin{array}{l}
\dot{x}=-k_{1} x y \\
\dot{y}=k_{1} x y-k_{2} y
\end{array}, k_{1}, k_{2}>0 .\right.
$$

The Lagrange and Hamilton functions of the system (4) are:

$$
\begin{gathered}
L=\frac{1}{2}\left(\frac{\ln y}{x} \dot{x}-\frac{\ln x}{y} \dot{y}\right)+k_{1}(x+y)-k_{2} \ln x, \\
H=\quad-k_{1}(x+y)+k_{2} \ln x
\end{gathered}
$$

and so we have a new conservation law for (4):

$$
H=E_{L}=-k_{1}(x+y)+k_{2} \ln x .
$$

If we get back to the Kermack-McKendrick model (3), then we have that the corresponding Lagrangian is $\bar{L}=L+\frac{1}{2}\left(\dot{z}-k_{1} y\right)^{2}$, where $L$ is the Lagrangian of the subsystem (4).

\section{Feedback Linearization Method}

One way of determining the stability of stationary points is to linearize the system (by taking partial derivatives) and determine the stability of these points in the linear system ([24]).

Let us consider the general case of affine systems of the form

$$
\dot{\mathbf{x}}=f(\mathbf{x})+u \cdot g(\mathbf{x}) .
$$


Equation (5) describes the evolution of a dynamical system in $\mathbf{R}^{n}$, in which $u$ is a real variable that represents the control. This system can be transformed into one that is time-invariant by using a coordinate transformation $([22])$.

If $T(\mathbf{x})$ is a diffeomorphism and $\mathbf{z}=T(\mathbf{x})$, then we have:

$$
\dot{\mathbf{z}}=\frac{\partial T}{\partial \mathbf{x}} \dot{\mathbf{x}}=\frac{\partial T}{\partial \mathbf{x}}[f(\mathbf{x})+u g(\mathbf{x})] .
$$

Since $T$ is a diffeomorphism, $T^{-1}$ exists and, knowing $\mathbf{z}$, we have:

$$
\mathbf{x}=T^{-1}(\mathbf{z}) \text {. }
$$

We look for a diffeomorphism $T: D \subseteq \mathbf{R}^{n} \longrightarrow \mathbf{R}^{n}$ defining the coordinate transformation

$$
\mathbf{z}=T(\mathbf{x})
$$

and a control law of the form

$$
u=\Phi(\mathbf{x})+\omega^{-1}(\mathbf{x}) v .
$$

These new variables are used to transform the system (5) into a new state space realisation as follows:

$$
\mathbf{Z}=A \mathbf{z}+v B
$$

Let us assume that, after the coordinate transformation (8), the system (5) takes the following form $([23])$ :

$$
\dot{\mathbf{z}}=A \mathbf{z}+\bar{\omega}(\mathbf{z})[u-\bar{\Phi}(\mathbf{z})] B=A \mathbf{z}+\omega(\mathbf{x})[u-\Phi(\mathbf{x})] B,
$$

where $\bar{\omega}(\mathbf{z})=\omega\left(T^{-1}(\mathbf{z})\right)$ and $\bar{\Phi}(\mathbf{z})=\Phi\left(T^{-1}(\mathbf{z})\right)$.

Substituting (6) and (8) into (11), we find that

$$
\frac{\partial T}{\partial \mathbf{x}}[f(\mathbf{x})+u g(\mathbf{x})]=A T(\mathbf{x})+\omega(\mathbf{x})[u-\Phi(\mathbf{x})] B .
$$

Equation (12) is satisfied if

$$
\left\{\begin{array}{lr}
\frac{\partial T}{\partial \mathbf{x}} f(\mathbf{x})=A T(\mathbf{x})-\omega(\mathbf{x}) \Phi(\mathbf{x}) B \\
\frac{\partial T}{\partial \mathbf{x}} g(\mathbf{x})= & \omega(\mathbf{x}) B
\end{array}\right.
$$

Remark. Assuming $(A, B)$ controllable, we can consider that $(A, B)$ are in the controllable form:

$$
A_{C}=\left(\begin{array}{ccccc}
0 & 1 & 0 & \cdots & 0 \\
0 & 0 & 1 & \cdots & 0 \\
\vdots & \vdots & \vdots & \vdots & \vdots \\
0 & 0 & 0 & \cdots & 0
\end{array}\right), B_{C}=\left(\begin{array}{c}
0 \\
0 \\
\vdots \\
1
\end{array}\right) \text {, }
$$

where $A_{C}$ is an $n \times n$ matrix, and $B_{C}$ is an $n \times 1$ matrix.

Letting

$$
T(\mathbf{x})=\left(\begin{array}{c}
T_{1}(\mathbf{x}) \\
T_{2}(\mathbf{x}) \\
\vdots \\
T_{n}(\mathbf{x})
\end{array}\right)
$$

with $A=A_{C}, B=B_{C}$ and $\mathbf{z}=T(\mathbf{x})$, the right-hand side of Equations (13) becomes

$$
A_{C} T(\mathbf{x})-\omega(\mathbf{x}) \Phi(\mathbf{x}) B_{C}=\left(\begin{array}{c}
T_{2}(\mathbf{x}) \\
T_{3}(\mathbf{x}) \\
\vdots \\
T_{n}(\mathbf{x}) \\
-\Phi(\mathbf{x}) \omega(\mathbf{x})
\end{array}\right)
$$




$$
\omega(\mathbf{x}) B_{C}=\left(\begin{array}{c}
0 \\
0 \\
\vdots \\
0 \\
\omega(\mathbf{x})
\end{array}\right) .
$$

Substituting Equations (16) and (17) into (14) and (15), we find, after some calculations, that the components $T_{1}, T_{2}, \ldots, T_{n}$ of the coordinate transformation $T$ must be as follows ([23]):

$$
\begin{aligned}
& \left\{\begin{array}{l}
\frac{\partial T_{i}}{\partial \mathbf{x}} g(\mathbf{x})=0, \quad i=1,2, \ldots n-1, \\
\frac{\partial T_{n}}{\partial \mathbf{x}} g(\mathbf{x}) \neq 0,
\end{array}\right. \\
& \frac{\partial T_{i}}{\partial \mathbf{x}} f(\mathbf{x})=T_{i+1}, \quad i=1,2, \ldots n-1 .
\end{aligned}
$$

The functions $\Phi$ and $\omega$ are given by

$$
\omega(\mathbf{x})=\frac{\partial T_{n}}{\partial \mathbf{x}} g(\mathbf{x}), \Phi=-\frac{\left(\frac{\partial T_{n}}{\partial \mathbf{x}}\right) f(\mathbf{x})}{\left(\frac{\partial T_{n}}{\partial \mathbf{x}}\right) g(\mathbf{x})} .
$$

\section{The Transformed 2D Kermack-McKendrick Model via the Feedback Linearization Method}

In this section, we consider the problem of finding a state feedback control for the $2 D$ Kermack-McKendrick system, in order to obtain a suitable form of this system.

Let us consider the Kermack-McKendrick model in the form ([9], [25]):

$$
\left\{\begin{array}{l}
\frac{\mathrm{d} S}{\mathrm{~d} t}=-\lambda S I, \\
\frac{\mathrm{d} I}{\mathrm{~d} t}=\lambda S I-\sigma I,
\end{array}\right.
$$

where $S$ represents the susceptible class, $I$ the infected class and $R$ the removed class, with $\frac{d R}{\mathrm{~d} t}=\sigma I$. The parameter $\sigma>0$ is the removal rate and $\lambda>0$ is the infection rate.

We assume that $S(0)=S_{0}$ and $I(0)=I_{0}$ are given. For the simplicity of the computation, let us denote $S=x, I=y$ and $\mathbf{x}=(x, y)$. The system (21) becomes

$$
\left\{\begin{array}{l}
\frac{\mathrm{d} x}{\mathrm{~d} t}=-\lambda x y, \\
\frac{\mathrm{d} y}{\mathrm{~d} t}=\lambda x y-\sigma y .
\end{array}\right.
$$

That is, in vector form:

$$
\left(\begin{array}{c}
\dot{x} \\
\dot{y}
\end{array}\right)=\left(\begin{array}{c}
0 \\
-\sigma y
\end{array}\right)+u \cdot\left(\begin{array}{c}
-\lambda x y \\
\lambda x y
\end{array}\right), u \in \mathbf{R},
$$

with $f, g: \mathbf{R}^{2} \longrightarrow \mathbf{R}^{2}, f(\mathbf{x})=\left(\begin{array}{c}0 \\ -\sigma y\end{array}\right), g(\mathbf{x})=\left(\begin{array}{c}-\lambda x y \\ \lambda x y\end{array}\right), \forall \mathbf{x}=(x, y) \in \mathbf{R}^{2}$.

According to the previous section, we look for a transformation $T=\left(T_{1}, T_{2}\right)^{t}$ such that the conditions

$$
\left\{\begin{aligned}
\frac{\partial T_{1}}{\partial x} g_{x}+\frac{\partial T_{1}}{\partial y} g_{y} & =0, \\
\frac{\partial T_{2}}{\partial x} g_{x}+\frac{\partial T_{2}}{\partial y} g_{y} & \neq 0 \\
\frac{\partial T_{1}}{\partial x}(0)+\frac{\partial T_{1}}{\partial y}(-\sigma y) & =T_{2}
\end{aligned}\right.
$$

are fulfilled. 
From the third relation in (24), we deduce that $T_{2}=-\sigma y \frac{\partial T_{1}}{\partial y}$, while the first one yields $\frac{\partial T_{1}}{\partial x}=\frac{\partial T_{1}}{\partial y}$. We obtain that

$$
\left\{\begin{array}{l}
T_{1}(x, y)=\lambda(x+y), \\
T_{2}(x, y)=-\sigma \lambda y
\end{array}\right.
$$

According to Section 3, the functions $\omega(\mathbf{x})$ and $\Phi(\mathbf{x})$ are as follows:

$$
\begin{gathered}
\omega(\mathbf{x})=\left(\frac{\partial T_{2}}{\partial x} \frac{\partial T_{2}}{\partial y}\right) g(\mathbf{x})=\left(\begin{array}{cl}
0 y-\lambda \sigma
\end{array}\right)\left(\begin{array}{c}
-\lambda x y \\
\lambda x y
\end{array}\right)=-\lambda^{2} \sigma x y, \\
\Phi(\mathbf{x})=-\frac{\left(\frac{\partial T_{2}}{\partial \mathbf{x}}\right) f(\mathbf{x})}{\left(\frac{\partial T_{2}}{\partial \mathbf{x}}\right) g(\mathbf{x})}=-\frac{\sigma}{\lambda} \cdot \frac{1}{x} .
\end{gathered}
$$

The new linearized system, in the new coordinates $\mathbf{z}=\left(z_{1}, z_{2}\right)$, will be:

$$
\dot{\mathbf{z}}=\left(\begin{array}{c}
\dot{z}_{1} \\
\dot{z}_{2}
\end{array}\right)=\left(\begin{array}{ll}
0 & 1 \\
0 & 0
\end{array}\right)\left(\begin{array}{l}
z_{1} \\
z_{2}
\end{array}\right)-\lambda^{2} \sigma z_{1} z_{2} \cdot\left(u+\frac{\sigma}{\lambda} \cdot \frac{1}{z_{1}}\right)\left(\begin{array}{l}
0 \\
1
\end{array}\right),
$$

with the controllers

$$
A_{C}=\left(\begin{array}{ll}
0 & 1 \\
0 & 0
\end{array}\right), \quad B_{C}=\left(\begin{array}{l}
0 \\
1
\end{array}\right) .
$$

The system (26) is equivalent to

$$
\left\{\begin{array}{l}
\dot{z}_{1}=z_{2} \\
\dot{z}_{2}=-\lambda^{2} \sigma z_{1} z_{2} u-\lambda^{2} \sigma^{2} z_{2}
\end{array}\right.
$$

Remarks. 1) The form of the transformed system (27) depends on the choice of the controllers $A_{C}$ and $B_{C}$.

2) Let us observe that the system (27), obtained by this method, has a specific form which is similar to the transformed form for the $2 D$ Lotka-Volterra system ([2]).

\section{Optimal Control of a Stochastic Version of the $2 D$ Kermack-McKendrick Model}

We consider the controlled system

$$
\begin{aligned}
& \dot{x}(t)=-\lambda x(t) y(t) u(t), \\
& \dot{y}(t)=-\sigma y(t)+\lambda x(t) y(t) u(t),
\end{aligned}
$$

where $\lambda$ and $\sigma$ are positive constants. If $u(t) \equiv 1$, this system is the (two-dimensional) Kermack-McKendrick model for the spread of epidemics.

From the previous section, we deduce that the feedback linearization of $(28),(29)$ is the following system:

$$
\begin{aligned}
& \dot{z}_{1}(t)=z_{2}(t) \\
& \dot{z}_{2}(t)=-\lambda^{2} \sigma^{2} z_{2}(t)-\lambda^{2} \sigma z_{1}(t) z_{2}(t) u(t) .
\end{aligned}
$$

Next, we consider a stochastic version of the transformed model:

$$
\begin{aligned}
& \dot{z}_{1}(t)=z_{2}(t), \\
& \dot{z_{2}}(t)=-\lambda^{2} \sigma^{2} z_{2}(t)-\lambda^{2} \sigma z_{1}(t) z_{2}(t) u(t)+\left[v\left(z_{1}(t), z_{2}(t)\right)\right]^{1 / 2} \dot{W}(t),
\end{aligned}
$$

where $v\left(z_{1}(t), z_{2}(t)\right)$ is a positive function, and $\{W(t), t \geq 0\}$ is a standard Brownian motion.

Let

$$
T(x, y):=\inf \{t \geq 0: y(t)=0 \mid x(0)=x, y(0)=y\} .
$$


That is, $T(x, y)$ is the first time the number of infected individuals is equal to zero. We can write that

$$
T(x, y)=\tau\left(z_{1}, z_{2}\right):=\inf \left\{t \geq 0: z_{2}(t)=0 \mid z_{1}(0)=z_{1}, z_{2}(0)=z_{2}\right\} .
$$

We consider the cost criterion

$$
J\left(z_{1}, z_{2}\right)=\int_{0}^{\tau\left(z_{1}, z_{2}\right)}\left\{-\frac{1}{2} q_{0} z_{1}(t) z_{2}(t) u^{2}(t)+\gamma\right\} \mathrm{d} t,
$$

where $q_{0}$ and $\gamma$ are positive constants. We look for the control $u(t)$ that minimizes the expected value of $J\left(z_{1}, z_{2}\right)$.

Remark. Because $\gamma$ is positive, the aim is to minimize the time it takes to cure all the infected individuals, taking the quadratic control costs into account, which is a natural and important problem to consider. As we mentioned in the Introduction section, this type of problem is known as LQG homing (see [28], [29], [30]). Except in special instances, it is usually very difficult to obtain analytical solutions to such problems in two or more dimensions. In this section, we will manage to derive the optimal solution to the problem set up above in a particular instance by making use of the method of similarity solutions.

Let $F\left(z_{1}, z_{2}\right)$ be the value function; that is,

$$
F\left(z_{1}, z_{2}\right):=\inf _{u(t), 0 \leq t \leq \tau\left(z_{1}, z_{2}\right)} E\left[J\left(z_{1}, z_{2}\right)\right] .
$$

Making use of dynamic programming, we find that $F$ is such that

$$
-\frac{1}{2} q_{0} z_{1} z_{2} u^{2}+\gamma+z_{2} F_{z_{1}}-\left(\lambda^{2} \sigma^{2} z_{2}\right) F_{z_{2}}-\left(\lambda^{2} \sigma z_{1} z_{2} u\right) F_{z_{2}}+\frac{1}{2} v\left(z_{1}, z_{2}\right) F_{z_{2} z_{2}}=0,
$$

where $u=u(0)$.

Differentiating Equation (38) with respect to $u$, we obtain that the optimal control is given by

$$
u^{*}=-\frac{\lambda^{2} \sigma}{q_{0}} F_{z_{2}} .
$$

Substituting this expression into (38), we obtain that $F$ satisfies the second-order nonlinear partial differential equation

$$
\frac{1}{2 q_{0}}\left(\lambda^{4} \sigma^{2}\right) z_{1} z_{2}\left(F_{z_{2}}\right)^{2}+\gamma+z_{2} F_{z_{1}}-\left(\lambda^{2} \sigma^{2} z_{2}\right) F_{z_{2}}+\frac{1}{2} v\left(z_{1}, z_{2}\right) F_{z_{2} z_{2}}=0 .
$$

The boundary condition is

$$
F\left(z_{1}, z_{2}\right)=0 \quad \text { if } z_{2}=0
$$

Moreover, we must have:

$$
\lim _{z_{2} \rightarrow-\infty} F\left(z_{1}, z_{2}\right)=\infty
$$

because if $z_{2}$ tends to $-\infty$, it will take an infinite time to bring $z_{2}(t)$ to zero (or the optimizer will need to use infinite control, which also leads to $\left.F\left(z_{1}, z_{2}\right)=\infty\right)$.

Next, assume that

$$
v\left(z_{1}, z_{2}\right)=-\sigma_{0}^{2} z_{1} z_{2}
$$

and let

$$
\Phi\left(z_{1}, z_{2}\right):=e^{-\alpha F\left(z_{1}, z_{2}\right)},
$$

where

$$
\alpha:=\frac{\lambda^{4} \sigma^{2}}{q_{0} \sigma_{0}^{2}}(>0) .
$$

We find that the function $\Phi\left(z_{1}, z_{2}\right)$ satisfies the second-order linear partial differential equation

$$
-\gamma \alpha \Phi+z_{2} \Phi_{z_{1}}-\lambda^{2} \sigma^{2} z_{2} \Phi_{z_{2}}-\frac{1}{2} \sigma_{0}^{2} z_{1} z_{2} \Phi_{z_{2} z_{2}}=0
$$


subject to

$$
\Phi\left(z_{1}, 0\right)=1 \quad \text { and } \quad \lim _{z_{2} \rightarrow-\infty} \Phi\left(z_{1}, z_{2}\right)=0 .
$$

Finally, let us try a solution of the form

$$
\Phi\left(z_{1}, z_{2}\right)=\Psi(z)
$$

where $z:=-z_{2} / z_{1}(\geq 0)$. We find that Equation (46) is transformed into the ordinary differential equation

$$
-\gamma \alpha \Psi+z^{2} \Psi^{\prime}-\lambda^{2} \sigma^{2} z \Psi^{\prime}+\frac{1}{2} \sigma_{0}^{2} z \Psi^{\prime \prime}=0
$$

From the definition of $z_{1}(t)$ and $z_{2}(t)$ in terms of $x(t)$ and $y(t)$, we find that

$$
z=0 \quad \text { if } z_{2}=0 \text { and } \quad \lim _{z_{2} \rightarrow-\infty} z=\sigma .
$$

Hence, the boundary conditions are

$$
\Psi(0)=1 \quad \text { and } \quad \Psi(\sigma)=0 .
$$

The mathematical software Maple gives an expression for the general solution of Equation (49), which is expressed in terms of the special function HeunB.

Let us consider the particular case when

$$
\lambda=\sigma=q_{0}=\gamma=\sigma_{0}=1
$$

We then have $\alpha=1$, and Equation (49) becomes

$$
-\Psi(z)+z^{2} \Psi^{\prime}(z)-z \Psi^{\prime}(z)+\frac{1}{2} z \Psi^{\prime \prime}(z)=0
$$

whose general solution can be written as follows:

$$
\Psi(z)=z \operatorname{HeunB}(1,-2,-1,4, z) e^{-z(z-2)}\left[c_{1}+c_{2} \int \frac{e^{z(z-2)}}{z^{2} \operatorname{HeunB}(1,-2,-1,4, z)^{2}} \mathrm{~d} z\right] .
$$

Let us define

$$
\Psi_{1}(z)=z \operatorname{HeunB}(1,-2,-1,4, z) e^{-z(z-2)}
$$

and

$$
\Psi_{2}(z)=z \operatorname{HeunB}(1,-2,-1,4, z) e^{-z(z-2)} \int_{z}^{1} \frac{e^{w(w-2)}}{w^{2} \operatorname{HeunB}(1,-2,-1,4, w)^{2}} \mathrm{~d} w .
$$

We find that $\Psi_{1}(0)=\Psi_{2}(1)=0$ and that $\lim _{z \downarrow 0} \Psi_{2}(z)=1$. Hence, we set $c_{1}=0$ and $c_{2}=1$, so that the solution that satisfies the above boundary conditions is $\Psi(z)=\Psi_{2}(z)$. This function is shown in Figure 1 . From the function $\Psi(z)$, we can calculate the optimal control $u^{*}=-F_{z_{2}}$.

\section{Conclusions}

The $2 D$ Kermack-McKendrick system admits a controllable form in the context of Section 4, and the new linearized system (27) is similar to the linearized form of the Lotka-Volterra system ([2]). This new controllable form has a significant change in the distribution of the system parameters. A further objective is to analyze and evaluate these linearized forms, in order to devise geometric methods for them. The results will be used in a qualitative and quantitative analysis of the behavior of the individuals in the framework of an epidemic that respects the mathematical model of Kermack and McKendrick, and also for further generalizations. The influence of the parameters will also be studied, as well as further possible relations with the Hamiltonian formalism. 


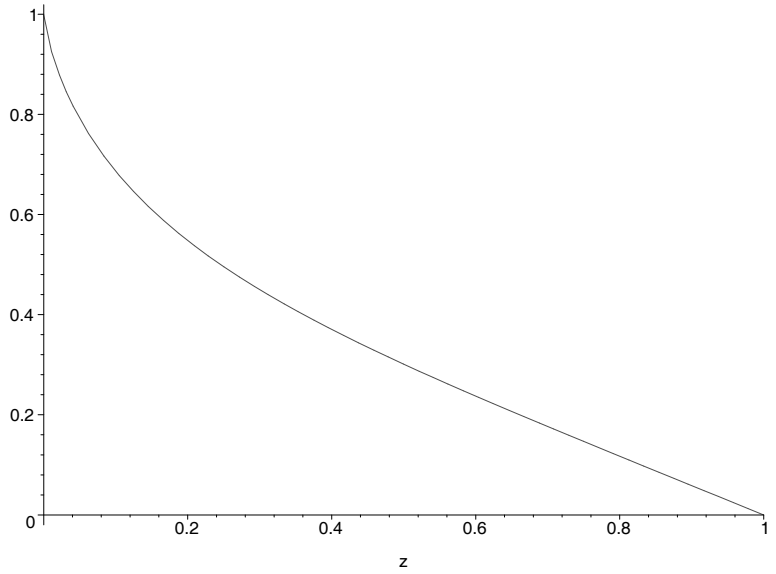

Figure 1. Function $\Psi(z)$.

In general, the Kermack-McKendrick model of epidemics can be useful in studying real world behavior. Excluding the cases in which unbounded growth of any population occurs, this model is applicable in the real world. By first understanding the exponential growth model, one can better understand why a logistic growth model would be more realistic. By studying the $2 D$ and $3 D$ systems, several useful techniques could be devised for the study of the stability of nonlinear dynamical systems.

Finally, in the case of the optimal control problem solved in Section 5, it would be interesting to obtain at least approximate solutions to problems for which we cannot make use of the method of similarity solutions.

\section{Funding}

This research was supported by grant FP7-PEOPLE-2012-IRSES-316338 and by the Natural Sciences and Engineering Research Council of Canada (NSERC).

Acknowledgments. The authors are grateful to the reviewer for his/her judicious remarks.

\section{References}

1. Ionescu A, Militaru R, Munteanu F. Geometrical methods and numerical computations for prey-predator systems, British J. of Math. Comp. Sci. 2015; 10 (5): 1-15.

2. Ionescu A, Munteanu F. On the feedback linearization for the 2D prey-predator dynamical systems, Bul. St. Univ. Politeh. Timişoara, Ser. Mat.-Fiz. 2015; 60 (74) (2): 17-25.

3. Graunt J. Natural and Political Observations Made Upon the Bills of Mortality, London; 1662.

4. Naz R, Naeem I, Mahomed FM. A partial Lagrangian approach to mathematical models of epidemiology, Mathematical Problems in Engineering 2015. Article ID 602915, 11 pages.

5. Bernoulli D. Essai d'une nouvelle analyse de la mortalité causée par la petite vérole et des avantages de l'inoculation pour la prévenir, Mém. Math. Phys. Acad. Roy. Sci., Paris; 1760, 145 pages.

6. Bernoulli D. Réflexions sur les avantages de l'inoculation, Mercure de France; 1760; 173-190.

7. Murray JD. Mathematical Biology, Springer-Verlag, New York; 2002.

8. Young T, Buckalew R, May A, Boczko E. A low dimensional dynamical model of the initial pulmonary innate response to infection, Mathematical Biosciences 2012; 235: 189âĂŞ-200.

9. Kermack WD, McKendrick AG. A contribution to the mathematical theory of epidemics, J. Royal Stat. Soc. 1927; A115: 700-721; 1932; A138: 55-83.

10. Bailey NTJ. The Mathematical Theory of Infectious Diseases, Hafner Press, New York; 1975.

11. Townsend CR, Harper JL, Begon M. Ecology: Individuals, Populations and Communities, Blackwell Sci. Publ., Oxford; 1990. 
12. Brauer F. Compartmental models in epidemiology, Mathematical Epidemiology, vol. 1945, Lecture Notes in Mathematics, Springer, Berlin, Germany; 2008; 19-79.

13. Lotka AJ. Elements of Physical Biology, Williams-Wilkins, Baltimore; 1925.

14. Volterra V. Leçons sur la Théorie Mathématique de la Lutte pour la Vie, Gauthier-Villars; 1931.

15. Volterra V. Principes de biologie mathématique, Acta Biother. 1937; 3: 1-36.

16. Militaru R, Munteanu F. Geometric methods for the study of biodynamical systems. Symmetries and conservation laws, Proc. of the Int. Conf. Cellular and Molecular Biology (WSEAS) Recent Researches in Medicine, Biology and Bioscience; 2013; 54-61.

17. Militaru R, Munteanu F. Symmetries and conservation laws for biodynamical systems, Int. J. of Math. Models and Meth. in Appl. Sci. 2013; 12 (7): 965-972.

18. Militaru R, Munteanu F. Computational analysis of conservation laws for prey-predators Lotka-Volterra systems, Proc. of Int. Conf. of Appl. and Theor. Electricity (ICATE), Craiova, October 23-25, 2014, Editor IEEE; 2014; $1-7$.

19. Militaru R, Munteanu F. Geometrical methods and numerical computations for the study of mathematical models of the multi-species interactions, BSGP 2015; 22: 51-60.

20. Munteanu F. A study of the dynamical systems in the Lagrangian and Hamiltonian formalism, Proc. of SSMR Conference, Braşov; 2001; 169-178.

21. Nutku Y. Hamiltonian structure of the Lotka-Volterra equations, Physics Letters A. 1990; 145 (1): 27-28.

22. Henson M, Seborg D. Nonlinear Process Control, Prentice Hall, Englewood Cliffs, New Jersey; 2005.

23. Isidori A. Nonlinear Control Systems, Springer-Verlag, New York; 1989.

24. Siburg KF. Gewöhnliche Differentialgleichungen (Ordinary Differential Equations), Lecture at the University of Dortmund, Germany. Spring-Summer Semester; 2005.

25. Obădeanu V. Differential Dynamical Systems. The Dynamics of Biological and Economical Processes (in Romanian), UVT Press, Timişoara; 2006.

26. Olver PJ. Applications of Lie Groups to Differential Equations, Graduate Texts in Mathematics, 107, SpringerVerlag, New York; 1986.

27. Whittle P, Optimization over Time, Vol. I, Wiley, Chichester; 1982.

28. Lefebvre M, Zitouni F. General LQG homing problems in one dimension, Int. J. Stoch. Anal. 2012, Article ID 803724, 20 pages. doi:10.1155/2012/803724

29. Lefebvre M, Zitouni F. Analytical solutions to LQG homing problems in one dimension, Systems Science and Control Engineering: An Open Access Journal 2014; 2: 41-47.

30. Makasu C. Explicit solution for a vector-valued LQG homing problem, Optim. Lett. 2013; 7: 607-612. 